\title{
In vitro cytocompatibility evaluation of collagen based scaffolds using human endothelial progenitor cells for vascular tissue engineering
}

https://doi.org/10.33263/BTEB114.010016

Florin Iordache ${ }^{1, *}$, Andrei Constantinescu ${ }^{1}$, Eugen Andrei ${ }^{1}$, Carmen Curuțiu ${ }^{2}$,

Alexandru Mihai Grumezescu ${ }^{3}$, Georgeta Voicu ${ }^{3}$, Horia Maniu ${ }^{1}$

1 Institute of Cellular Biology and Pathology "Nicolae Simionescu, of Romanian Academy, Department of Fetal and Adult Stem Cell Therapy, 050568, Bucharest, Romania

2 University of Bucharest, Faculty of Biology, Department of Microbiology-Immunology, Bucharest, Romania

3 Politehnica University of Bucharest, Faculty of Applied Chemistry and Material Science, 011061, Bucharest, Romania

*corresponding author e-mail address: floriniordache84@yahoo.com

\section{ABSTRACT}

Vascular tissue engineering attempts to grow blood vessels through the use of different scaffolds that allows vascular cells such as endothelial cells to form networks and organized in vascular tissue. Various biomaterials are used to produce scaffolds that allow growth and differentiation of stem cells; depending on the cell type and applications some materials are more suitable than other. The aim of this study was to evaluate the cytocompatibility of collagen based scaffolds and to assess the capacity of endothelial progenitor cells (EPC) isolated from human umbilical cord to form vascular networks on these scaffolds. Our results show that after 5 days in culture with collagen scaffolds, the EPC remained viable, a sign of biocompatibility with the 3D scaffolds. Scanning electron microscopy showed that in the collagen scaffolds EPC organize within networks and presents an abundant extracellular matrix that strengthen the links between them. When EPC were cultured on collagenchitosan scaffolds, they are more adherent to the scaffolds compared with collagen, exibiting a good capacity to form networks. This study shows that the collagen and collagen-chitosan scaffolds are not cytotoxic for EPC and they provide the possibility of being used in vascular tissue engineering to help creating blood vessels.

Keywords: collagen, chitosan, matrigel, biocompatibility, stem cells, vascular tissue engineering.

\section{INTRODUCTION}

Different types of materials including biodegradable synthetic polymers, decellularized xenogenous tissues, and natural materials have been used in vascular tissue engineering (Sell et al., 2009). Biodegradable synthetic polymers are limited due to inflammation and immune response, relatively fast degradation rate, and instability in different environments. Therefore recent studies focus on the development of natural biomaterials for development of blood vessels (Deng et al., 2010; Parida et al., 2012; Subramanian et al., 2009). Collagens are the most abundant mammalian proteins and serves as adhesion proteins that enhance cell attachment and proliferation through specific interactions between RGD (Arg-Gly-Asp) domains in collagen molecules and integrin receptors in the cell membrane (Constantinescu et al., 2014; Kim et al., 2014; Zhu et al., 2014). Collagens also can be used as matrix to explore the role of cytokines and growth factors in tube morphogenesis and sprouting (Hung et al., 2014). In addition to collagen, chitosan and its derivates are other biomaterials widely used in tissue engineering, for the repair of cartilage, liver, nerve, and skin (Parida et al., 2012). Due to their superior properties in biocompatibility, biodegradability, low-toxicity, and low antigenicity, chitosan scaffolds can function as growth factors delivery carriers to accelerate the tissue and blood vessel regeneration (Huang et al., 2014; Parida et al., 2012; Yang et al., 2010). Based on the cationic nature of the chitosan polymer, an ionic complex 
can be formed between chitosan and specific bioactive compounds. The temporary polyelectrolyte complex networks formed without covalent cross-linkers are more biocompatible, sensitive, and easily controlled and render chitosan a competent material for loading and releasing bioactive factors in a precisely controlled manner (Koc et al., 2014; Sheridan et al., 2014; Wang et al., 2013; Zhou et al., 2014).

Cell sources for vascular tissue engineering can be divided into somatic cells, progenitor and stem cells. Mature vascular cells like endothelial and muscular cells are terminally differentiated with limited proliferation capacity and thus limited expansion ability. EPC have more proliferation potential and plasticity to differentiate down a specific lineage. EPC from human umbilical cord blood participates to angiogenesis by forming new vascular networks and also secreting factors that promote vascular expansion in embryo (Lupu et al., 2011a; Lupu et al., 2011b). This type of cells could be a great source of vascular cells for development of blood vessels if aided by biocompatible scaffolds. There are many approaches that attempt to enhance the vascularization such as (i) adjusting the pore size of the scaffold; (ii) incorporation of glycosaminoglycans; (iii) adding angiogenic factors (VEGF, bFGF, PDGF, TGF, angiogenin), all these mechanism being controlled by the biomaterials properties (Deng et al., 2010; Koc et al., 2014; Wang et al., 2013). The aim of this study was to evaluate if collagen and collagen-chitosan scaffolds are biocompatible with EPC and permit the formation of vascular networks by compared with collagen basement matrix Matrigel.

\section{MATERIALS AND METHODS}

\section{Fabrication of collage and collagen-chitosan porous scaffolds}

Collagen (300.000 Da) gel was obtained in the Leather and Footwear Research InstituteCollagen Department starting from calf hides by chemical and enzymatic extraction. The collagen gel concentration was $2.54 \%$ and $\mathrm{pH}=7$. Cells were grown on 2 types of scaffolds: (i) crosslinked collagen (Col) and (ii) crosslinked collagenchitosan (Col-Chitosan). The scaffolds were prepared as follow: $50 \mathrm{~mL}$ of chitosan gel (2,54\%) was added drop by drop onto collagen gel $(50 \mathrm{~mL}$; $2,54 \%)$, under vigorous mixing and allowed to interact $30 \mathrm{~min}$. For the scaffolds that contain only collagen, in the synthesis step, the Chitosan was removed. After that, each hydrogel (Col, ColChitosan) was crosslinked in $1 \%$ glutaraldehyde, kept overnight at $5{ }^{\circ} \mathrm{C}$ and then lyophilized.

\section{Cell culture model}

The isolation and differentiation of EPCs was performed as previously described. Briefly, human umbilical cord blood (UCB) samples were collected at term delivery by specialized staff at the "Polizu" Hospital, Bucharest, Romania with informed consent, according to EU and Romanian legislation on the collection and handling of human biological samples and personal data protection. Mononuclear cells (MNC) were obtained by Histopaque (Sigma-Aldrich, Saint Louis, MO, USA) density gradient centrifugation at $400 \mathrm{~g}$, for 30 minutes at room temperature. After centrifugation, the MNC layer was harvested and washed twice in Dulbecco's Modified Eagle's Medium (DMEM, Sigma-Aldrich St. Louis, MO, USA) supplemented with $10 \%$ fetal bovine serum (FBS). The MNCs were plated on plastic dishes coated with fibronectin (1 $\mu \mathrm{g} / \mathrm{cm}^{3}$, Biosciences, San Jose, CA, USA) in endothelial differentiation EBM2 medium (Lonza, Switzerland), supplemented with 15\% FBS, 40 $\mathrm{ng} / \mathrm{mL}$ VEGF, $100 \mu \mathrm{g} / \mathrm{mL}$ endothelial cell growth supplement, $100 \mathrm{U} / \mathrm{mL}$ penicillin, $100 \mu \mathrm{g} / \mathrm{ml}$ streptomycin, and $50 \mu \mathrm{g} / \mathrm{mL}$ neomycin (SigmaAldrich, MO, USA). Cell cultures were maintained at $37^{\circ} \mathrm{C}$ with $5 \% \mathrm{CO}_{2}$ and $21 \% \mathrm{O}_{2}$ in a humidified atmosphere. On day one after plating, the nonadherent cells were discarded and fresh medium was applied. To maintain optimal culture conditions, medium was changed twice a week. For the proposed studies, were used cells from passage 8 . 


\section{Matrigel vascular tubes assay}

To evaluate the formation of vascular tubes in Matrigel, EPC were seeded into 96-well plates at a density of 3000 cells per well. Briefly, $50 \mu \mathrm{L}$ of Matrigel (Sigma-Aldrich, Saint Louis, MO, USA) was added in 96-well plates, and allowed to solidify for $30 \mathrm{~min}$ at $37^{\circ} \mathrm{C}$. After Matrigel polymerization, the cell suspension was added and incubated for 4-6 hours. Tube formation was observed using Eclipse TE300 microscope (Nikon, Tokyo, Japan).

\section{MTT viability assay}

Biocompatibility of EPC on collagen-based scaffolds was evaluated by MTT assay (CellTiter96 Non-Radioactive Cell Proliferation Assay kit, Promega, Madison, USA). EPCs were seeded in 24well plate, at a density of $5 \times 10^{4}$ cells/well, in DMEM medium, supplemented with $10 \%$ FBS. The cells suspension was added over collagen scaffolds (Col and Col-Chitosan) and incubated for 72 hours. Cell viability was performed at different time intervals (24-72h), according to the manufacturer's guidelines. Briefly, $15 \mu \mathrm{L}$ of Solution I was added in each well and incubated for 4 hours. Then, $100 \mu \mathrm{L}$ of Solution II was added to the 96-well plate, incubated for another hour and spectrophotometry measurements were performed at $570 \mathrm{~nm}$ using Mithras LB 940 (Berthold Technology, Germany).

\section{Fluorescent microscopy}

Biocompatibility of EPC with collagen-based scaffolds was assessed by fluorescent microscopy using RED CMTPX fluorophore (Life Technologies, Invitrogen, USA), a cell tracker for long-term tracing of living cells. The RED CMTPX dye was added to the culture medium at a final concentration of $5 \mu \mathrm{M}$, and incubated for 30 minutes to allow the dye to be up-taken by the cells. Then, the cells were washed with PBS and visualized by fluorescent microscopy. The nuclei were counterstained with $1 \mathrm{mg} / \mathrm{mL}$ DAPI $(4,6-$ diamidino-2 phenylindole). Photomicrographs were taken with a digital camera driven by the Axio-Vision 4.6 (Carl Zeiss, Germany) software.

\section{Scanning Electron Microscopy}

In order to observe the behavior of EPC on collagen-based scaffolds, scanning electron microscopy was used. EPC were grown on Col and Col-Chitosan for 72 hours then were washed with PBS and fixed in $2.5 \%$ glutaraldehyde for 1 hour at room temperature. Glutaraldehyde was then removed, and successive dehydration was carried out in ethanol $(40 \%, 60 \%, 70 \%, 80 \%, 90 \%$, and $100 \%)$ for 10 minutes each. SEM analysis was performed on a HITACHI S2600N electron microscope, at $25 \mathrm{keV}$, on samples covered with a thin silver layer.

\section{Statistical analysis}

Data were expressed as means \pm standard deviation (SD) where $n$ represents the number of experiments. Statistical analysis was performed by one-way ANOVA test; $P<0.05$ was considered statistically significant.

\section{RESULTS AND DISCUSSIONS}

Since their initial discovery, EPC have raised great enthusiasm given their therapeutic promises in a variety of disorders including cardiovascular disease, burn wounds, diabetes, and atherosclerosis. Flow cytometry analysis showed an endothelial phenotype, umbilical cord blood derived cells being positive for CD31, CD34, CD105, CD133, CD144, VEGFR2 surface markers (data not shown). The cells that we isolated from umbilical cord blood started developing after approximately 2 weeks in cultures and exhibited an epithelial-like phenotype, forming colonies of adherent cells with a cobblestone shape (Fig. 1a). The cells have the ability to form vascular networks on Matrigel matrix after 4 hour of cultivation (Fig. 1b) suggesting that they have the potential to differentiate in vascular cells. Matrigel basement matrix is most widely used to study angiogenesis in vitro; varieties of endothelial cells form capillarylike structures when are plated on top of this extracellular matrix scaffold.

The viability and proliferation of cells tested by MTT assay revealed that EPC were metabolic active in the presence of collagen and collagenchitosan scaffolds. A slight but significantly increase in proliferation could be observed in EPC cultured on collagen-based scaffolds (Fig. 2), suggesting that these scaffolds are not cytotoxic 
for EPC. To test the biocompatibility of EPC on collagen scaffolds the cells were labeled with RED CMTPX cell tracker that in the presence of viable cells become fluorescent (red) due to the activity of mitochondrial enzymes that activate the dye.
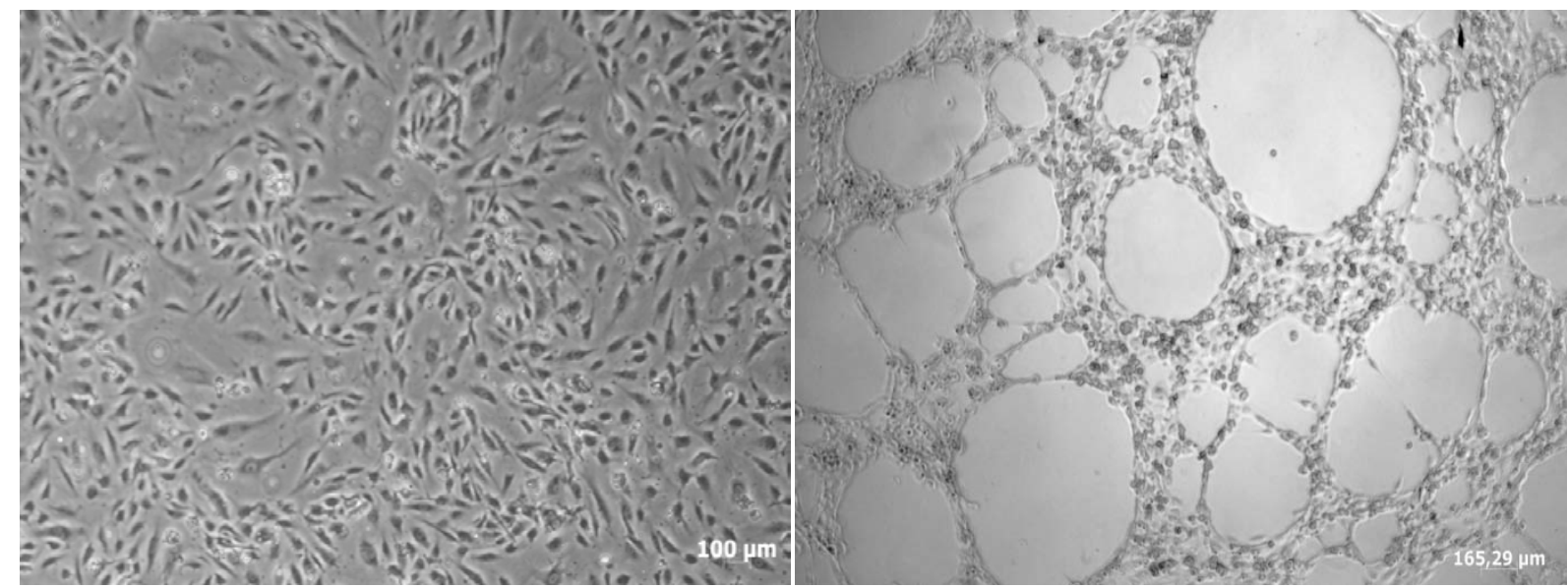

Figure 1. Morphology of EPC culture on fibronectin plate and on Matrigel basement membrane matrix.

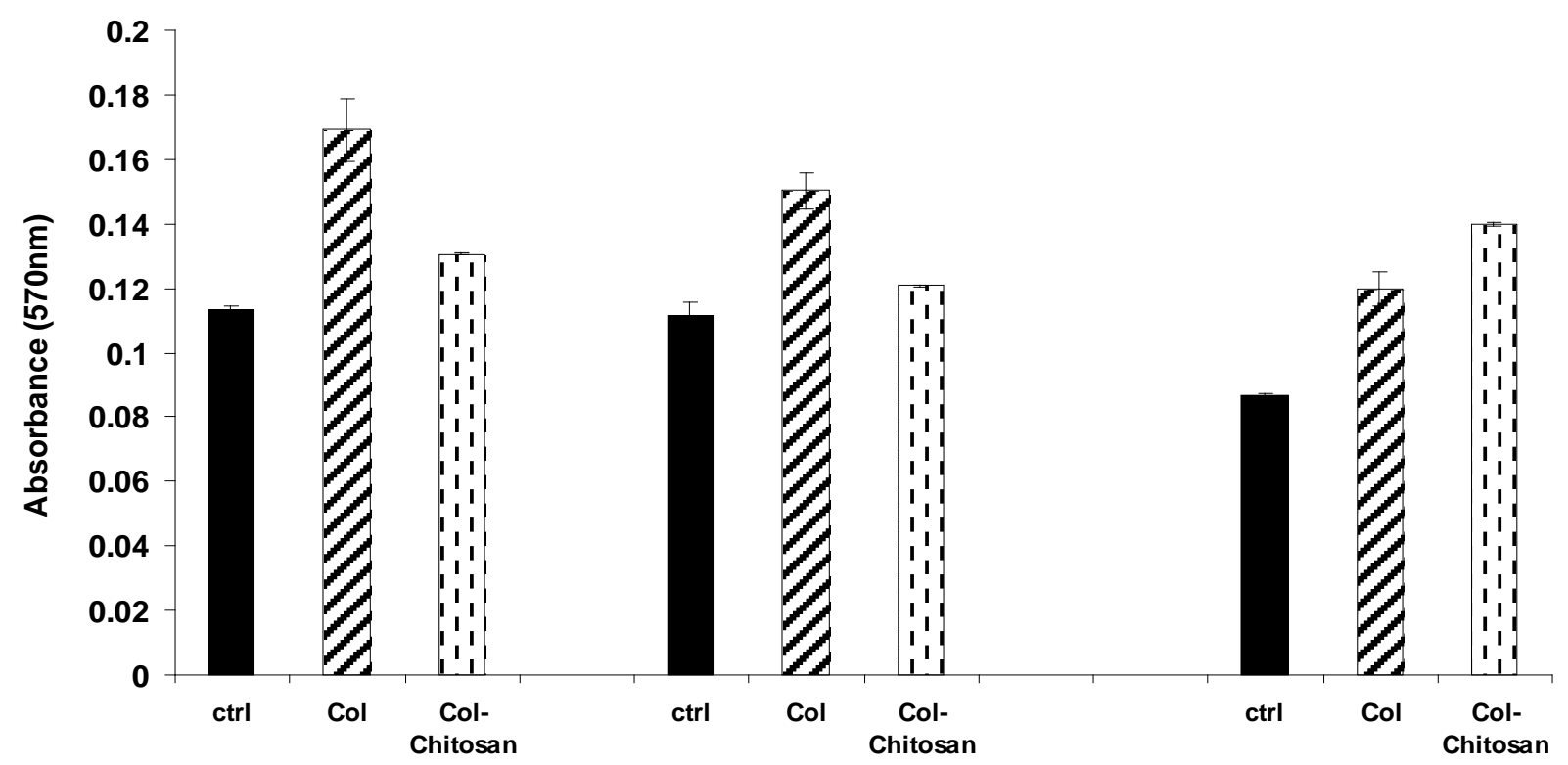

Figure 2. EPC viability on collagen based scaffolds. MTT test demonstrate no significant difference in viability of EPC cultured on collagen and collagen-chitosan compare with cell only. Results are represented as mean \pm standard error, $n=3,{ }^{*} p<0.05$.

Our results showed that after 5 days in culture with collagen-based scaffolds, the EPC are viable, morphologically intact, the fluorescence of the EPC culture on collagen-based scaffolds was comparable with control cells (Fig. 3a-c). Furthermore, SEM demonstrated that EPC adhered and spread on collagen and collagen-chitosan scaffolds demonstrating the biocompatibility with the 3D scaffolds. In the presence of Col (Fig. 4a) and Col-Chitosan (Fig. 4d) scaffolds, EPC become fusiform and emit filopodia that allow cells to interact each other and with the collagen and chitosan fibers, as it shown in Figure 4. In collagen scaffold the porous surface and irregular topography enable EPC to established links between cells and the scaffolds (Fig $4 b-c$ ). When EPC were cultured on collagen-chitosan scaffolds, they were more adherent compared with collagen scaffolds, exhibiting a better capacity to form networks (Fig 4e-f).

Vascular tissue engineering is an interdisciplinary field that applies the principles of engineering and life sciences towards the development of functional substitutes for damaged of vascular tissues (Deng et al., 2010). Blood vessel diseases such as atherosclerosis, 
chronic venous insufficiency and thrombosis remain the major cardiovascular diseases in the modern society (Lupu et al., 2011b). Materials for vascular replacements should be biomimetic meaning not causes thrombosis, inflammation, and permit neointimal proliferation of endothelial cells, and for all intents and purposes, they should resemble the native vessels. Therefore it is necessary to investigate the physical, chemical, and biological properties and modifications of materials to further understand the molecular mechanism of the cell-material interaction (Constantinescu et al., 2014). The formation of a microvasculature within a tissue-engineered organ or tissue will depend on multiple factors: the biochemical environment, endothelial cells type, the micro-architecture presented by the scaffold material, and mechanical signals (Huang et al., 2014).
Recent studies showed that collagen scaffolds create an environment that permits differentiation of EPC to adult vascular cells. Molecular biology data demonstrated that cultured EPC on collagen scafolds secrete various proangiogenic cytokines such as VEGF, endothelial nitric oxide synthase (eNOS), inducible nitric oxide synthase (iNOS), insulin-like growth factor 1 (IGF-1), stromalderived factor 1 (SDF-1), hepatocyte growth factor (HGF), and granulocyte colony-stimulating factor (G-CSF). The indirect contribution of EPCs to neovessel formation indicates that the cells can act as a source of proangiogenic cytokines to induce vascularization of tissue-engineered organoids in vitro (Deng et al., 2010; Wang et al., 2013). Our results showed that collagen and collagenchitosan scaffolds support the growth and proliferation of EPC, and also allow the cells to express their physiological role to form vascular networks and contribute to angiogenesis.
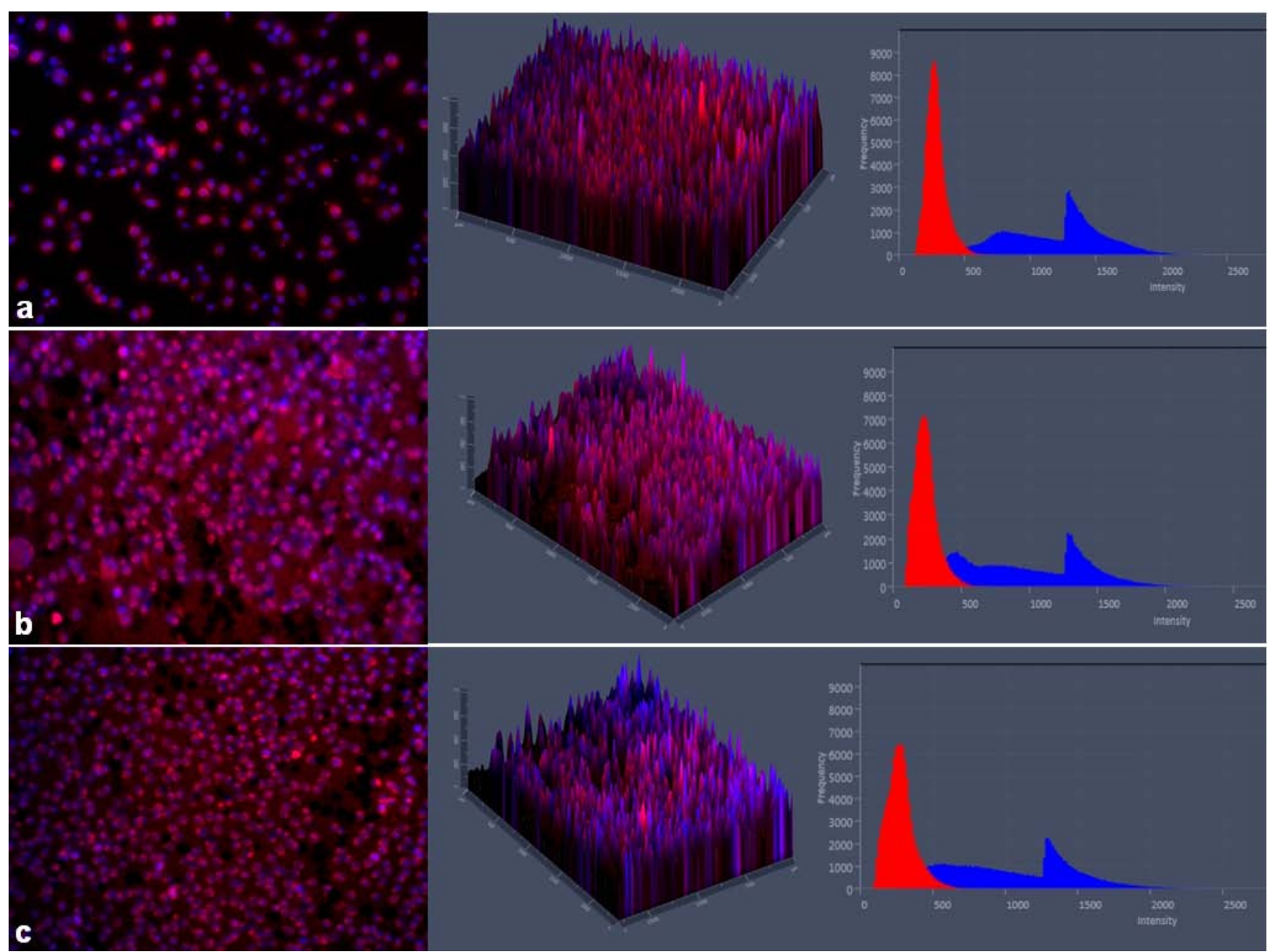

Figure 3. Photomicrographs of EPC labeled with vital RED CMTPX cell tracker in the presence of collagen (b) and (c) collagen-chitosan scaffolds, shows that cells are viable compared with control cells (a). The levels of fluorescent demonstrate no significant differences between cells only and cells culture on collagen-based scaffolds. Nuclei were counterstained with DAPI (1mg/mL). 


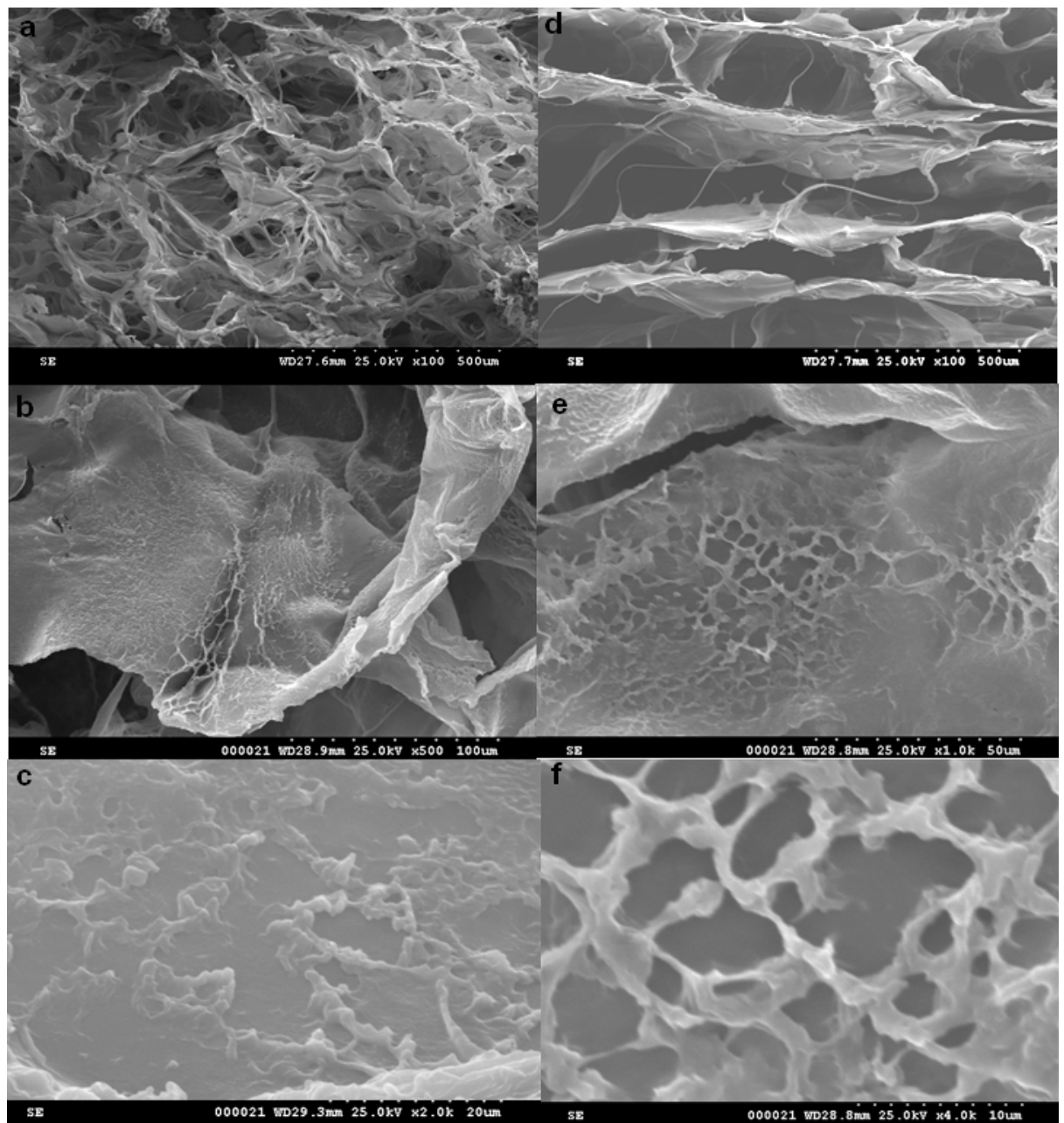

Figure 4. SEM photomicrographs of EPC cultured on collagen based-scaffolds. Collagen scaffolds seeded with EPC that attached and interacted with each other, forming network structures (a-c). EPC seeded on Collagen-chitosan scaffolds showed the ability to form complex networks, more tight and abundant (d-f).

\section{CONCLUSIONS}

Our results showed that collagen-based scaffolds appear to be ideally designed for vascular tissue engineering applications in terms of biocompatibility and properties to promote and support EPC growth and proliferation. From this morphological study, it appeared that EPCs were more adherent to collagen-chitosan scaffolds and formed tight networks compared with collagen scaffolds, rendering chitosan a suitable alternative for vascular tissue engineering.

\section{REFERENCE}

Constantinescu, A., Andrei, E., Iordache, F., Constantinescu, E., and Maniu, H. (2014). Recellularization potential assessment of Wharton's Jelly-derived endothelial progenitor cells using a human fetal vascular tissue model. In vitro cellular \& developmental biology Animal 50, 937-944.

Deng, C., Zhang, P., Vulesevic, B., Kuraitis, D., Li, F., Yang, A.F., Griffith, M., Ruel, M., and Suuronen, E.J. (2010). A collagen-chitosan hydrogel for endothelial differentiation and angiogenesis. Tissue engineering Part A 16, 3099-3109.

Huang, K.-F., Hsu, W.-C., Hsiao, J.-K., Chen, G.-S., and Wang, J.-Y. (2014). Collagen-Glycosaminoglycan Matrix Implantation Promotes Angiogenesis following Surgical Brain Trauma. BioMed Research International 2014, 12.

Hung, H.S., Chang, C.H., Chang, C.J., Tang, C.M., Kao, W.C., Lin, S.Z., Hsieh, H.H., Chu, M.Y., Sun, W.S., and 
Hsu, S.H. (2014). In Vitro Study of a Novel NanogoldCollagen Composite to Enhance the Mesenchymal Stem Cell Behavior for Vascular Regeneration. Plos One 9.

Kim, H.D., Heo, J., Hwang, Y., Kwak, S.Y., Park, O.K., Kim, H., Varghese, S., and Hwang, N.S. (2014). Extracellular-Matrix-Based and Arg-Gly-AspModified Photopolymerizing Hydrogels for Cartilage Tissue Engineering. Tissue engineering Part $A$.

Koc, A., Finkenzeller, G., Elcin, A.E., Stark, G.B., and Elcin, Y.M. (2014). Evaluation of adenoviral vascular endothelial growth factor-activated chitosan/hydroxyapatite scaffold for engineering vascularized bone tissue using human osteoblasts: In vitro and in vivo studies. J Biomater Appl 29, 748760.

Lupu, M., Khalil, M., Andrei, E., Iordache, F., Pfannkuche, K., Neef, K., Georgescu, A., Buzila, C., Brockmeier, K., Maniu, H., et al. (2011a). Integration Properties of Wharton's Jelly-derived Novel Mesenchymal Stem Cells into Ventricular Slices of Murine Hearts. Cell Physiol Biochem 28, 63-76.

Lupu, M., Khalil, M., Iordache, F., Andrei, E., Pfannkuche, K., Spitkovsky, D., Baumgartner, S., Rubach, M., AbdelRazik, H., Buzila, C., et al. (2011b). Direct contact of umbilical cord blood endothelial progenitors with living cardiac tissue is a requirement for vascular tube-like structures formation. J Cell Mol Med 15, 1914-1926.

Parida, P., Utkal University, B.O., Behera, A., Indian Institute of Technology, K., Chandra Mishra, S., and National Institute of Technology, R.O. (2012). Classification of Biomaterials used in Medicine. International Journal of Advances in Applied Sciences 1, 125-129.

Sell, S.A., McClure, M.J., Garg, K., Wolfe, P.S., and Bowlin, G.L. (2009). Electrospinning of collagen/biopolymers for regenerative medicine and cardiovascular tissue engineering. Adv Drug Deliver Rev 61, 1007-1019.

Sheridan, W.S., Grant, O.B., Duffy, G.P., and Murphy, B.P. (2014). The application of a thermoresponsive chitosan/beta-GP gel to enhance cell repopulation of decellularized vascular scaffolds. Journal of biomedical materials research Part B, Applied biomaterials 102, 1700-1710.

Subramanian, A., Krishnan, U.M., and Sethuraman, S. (2009). Development of biomaterial scaffold for nerve tissue engineering: Biomaterial mediated neural regeneration. Journal of biomedical science $16,108$.

Wang, L., Li, C.Y., Chen, Y.X., Dong, S.J., Chen, X.S., and Zhou, Y.M. (2013). Poly(lactic-co-glycolic) Acid/Nanohydroxyapatite Scaffold Containing Chitosan Microspheres with Adrenomedullin Delivery for Modulation Activity of Osteoblasts and Vascular Endothelial Cells. Biomed Research International.

Yang, W.J., Fu, J., Wang, D.X., Wang, T., Wang, H., Jin, S.G., and He, N.Y. (2010). Study on Chitosan/Polycaprolactone Blending Vascular Scaffolds by Electrospinning. J Biomed Nanotechnol 6, 254-259.

Zhou, M., Qiao, W., Liu, Z., Shang, T., Qiao, T., Mao, C., and Liu, C. (2014). Development and in vivo evaluation of small-diameter vascular grafts engineered by outgrowth endothelial cells and electrospun chitosan/poly(epsilon-caprolactone) nanofibrous scaffolds. Tissue engineering Part A 20, 79-91.

Zhu, C.H., Ma, X.X., Xian, L., Zhou, Y., and Fan, D.D. (2014). Characterization of a co-electrospun scaffold of HLC/CS/PLA for vascular tissue engineering. BioMed Mater Eng 24, 1999-2005.

\section{ACKNOWLEDGEMENTS}

This work was supported by the European Union and Ministry of Education - Scientific Research, Technological Development, POSCCE-A2-0.2.2.1-2013-1, grant "SORTIS" 649/1.04.2014.

\section{Conflicts of Interest}

The authors declare no conflict of interest.

(C) 2014 by the authors; licensee AMG Transcend, Bucharest, Romania. This article is an open access article distributed under the terms and conditions of the Creative Commons Attribution license (http://creativecommons.org/licenses/by/4.0/). 